\title{
Measurements of very forward particles production spectra at LHC: the LHCf experiment
}

\author{
E. Berti ${ }^{*}{ }^{a b}$ O. Adriani, ${ }^{a b}$ L. Bonechi, ${ }^{a}$ M. Bongi, ${ }^{a b}$ G. Castellini, ${ }^{a c}$ R. D'Alessandro, ${ }^{a b}$ \\ M. Haguenauer, ${ }^{d}$ Y. Itow, ${ }^{e f}$ T. Iwata,${ }^{g}$ K. Kasahara,${ }^{g}$ Y. Makino, ${ }^{e}$ K. Masuda,${ }^{e}$ \\ E. Matsubayashi, ${ }^{e}$ H. Menjo, ${ }^{h}$ Y. Muraki, ${ }^{e}$ P. Papini, ${ }^{a}$ S. Ricciarini, ${ }^{a c}$ T. Sako, ${ }^{e f}$ \\ T. Suzuki, ${ }^{g}$ T. Tamura,${ }^{j}$ A. Tiberio, ${ }^{a}{ }^{b}$ S. Torii, ${ }^{g}$ A. Tricomi, ${ }^{k l}$ W. C. Turner, ${ }^{m}$, M. Ueno, ${ }^{e}$ \\ Q. D. Zhou ${ }^{e}$
}

${ }^{a}$ INFN Section of Florence - Florence, Italy

${ }^{b}$ University of Florence - Florence, Italy

${ }^{c}$ IFAC-CNR - Florence, Italy

${ }^{d}$ Ecole-Polytechnique - Palaiseau, France

${ }^{e}$ Institute for Space-Earth Environmental Research - Furo-cho, Chikusa-ku, Nagoya, Japan

${ }^{f}$ Kobayashi-Maskawa Institute for the Origin of Particles and the Universe, Nagoya University Nagoya, Japan

${ }^{g}$ RISE, Waseda University - Shinjuku, Tokyo, Japan

${ }^{h}$ Graduate School of Science, Nagoya University - Nagoya, Japan

${ }^{i}$ CERN - Geneva, Switzerland

${ }^{j}$ Kanagawa University - Kanagawa, Japan

${ }^{k}$ INFN Section of Catania - Catania, Italy

${ }^{l}$ University of Catania - Catania, Italy

${ }^{m}$ LBNL - Berkeley, California, USA

E-mail: eugenio.berti@fi.infn.it

\begin{abstract}
Thanks to two small sampling calorimeters installed in the LHC tunnel at $\pm 140 \mathrm{~m}$ from IP1, the LHC forward (LHCf) experiment is able to detect neutral particles produced by high energy proton-ion collisions in the very forward region (pseudo-rapidity $\eta>8.4$ ). The main aim of LHCf is to provide precise measurements of the production spectra relative to these particles, in order to tune hadronic interaction models used by ground-based cosmic rays experiments. In this paper we will present the current status of the LHCf experiment, regarding in particular collected data and analysis results, as well as future prospects.
\end{abstract}

PACS : 13.85.-t, $13.85 . \mathrm{Tp}$

Fourth Annual Large Hadron Collider Physics

13-18 June 2016

Lund, Sweden

* Speaker. 


\section{Introduction}

In order to understand the processes responsible for acceleration and propagation of cosmic rays in the universe, measurements of their flux and composition up to the Greisen-ZatsepinKuzmin cut-off (GZK cut-off) are necessary. These measurements are performed by ground-based experiments through the indirect detection of the extensive air showers (EASs) that cosmic rays form when interacting with the atmosphere. The properties of the primary particle are then reconstructed making us of MC simulations that, being EASs physics described by soft (non perturbative) QCD, necessarily rely on phenomenological models. Among them very different predictions are found at high energies, due to the lack of experimental calibration data, resulting in large systematic uncertainties in cosmic rays measurements. The main purpose of the LHC forward (LHCf) experiment is to provide important information for the calibration of hadronic interaction models in the forward region. The Large Hadron Collider (LHC) is the most suitable place where to perform these measurements, because a center of mass energy of $\sqrt{s}=13 \mathrm{TeV}$ in p-p collisions is equivalent to about $9 \times 10^{16} \mathrm{eV}$ in the reference system where the target is at rest, an energy not so distant from the one of Ultra High Energy Cosmic Rays (UHECRs).

\section{The experiment}

LHCf [1] consists of two small sampling calorimeters installed in the Large Hadron Collider (LHC) tunnel at $\pm 140 \mathrm{~m}$ from IP1 (ATLAS interaction point). Being placed after the D1 dipole magnet, only neutral particles produced by proton-ion collisions and having pseudo-rapidity $\eta>$ 8.4 can reach the experiment. Each one of the two detectors, called Arm1 and Arm2, is made up by two square towers of $22 \mathrm{~W}$ and $16 \mathrm{GSO}$ (plastic scintillator before 2014 upgrade) layers for a total length of $29 \mathrm{~cm}$, equivalent to $44 X_{0}$ and $1.6 \lambda_{I}$. Towers size is $20 \mathrm{~mm} \times 20 \mathrm{~mm}$ and $40 \mathrm{~mm} \times 40$ $\mathrm{mm}$ for Arm1, $25 \mathrm{~mm} \times 25 \mathrm{~mm}$ and $32 \mathrm{~mm} \times 32 \mathrm{~mm}$ for Arm2. Energy resolution is better than $5 \%$ for $\gamma_{\mathrm{s}}$ above $100 \mathrm{GeV}$ and about $40 \%$ for hadrons above $500 \mathrm{GeV}$. The transverse position of the incident particle is reconstructed using 4 xy imaging layers inserted at different depths. They are formed by $1 \mathrm{~mm}$ width GSO-bars (scintillating fibers before 2014 upgrade) in the case of Arm 1 and by $160 \mu \mathrm{m}$ read-out pitch silicon microstrip detectors in the case of Arm2. Position resolution is better than $200 \mu \mathrm{m}$ for $\gamma_{\mathrm{s}}$ above $100 \mathrm{GeV}$ and $1 \mathrm{~mm}$ for hadrons above $500 \mathrm{GeV}$. More detailed descriptions of the detector are reported elsewhere [2,3].

\section{Analysis results}

Because LHCf requires low luminosity and high $\beta^{*}$, so far data have been acquired during special runs: in 2009-2010 p-p collisions at $\sqrt{s}=0.9$ and $7 \mathrm{TeV}$, in $2013 \mathrm{p}-\mathrm{p}$ at $\sqrt{s}=2.56 \mathrm{TeV}$ and p-Pb at $\sqrt{s_{N N}}=5.02 \mathrm{TeV}$, in $2015 \mathrm{p}-\mathrm{p}$ at $\sqrt{s}=13 \mathrm{TeV}$. While the analysis of this last data set is still ongoing, we will discuss here the results published so far, relative to $\gamma_{\mathrm{s}}, \pi^{0} \mathrm{~s}$ and neutrons produced in the forward region. 

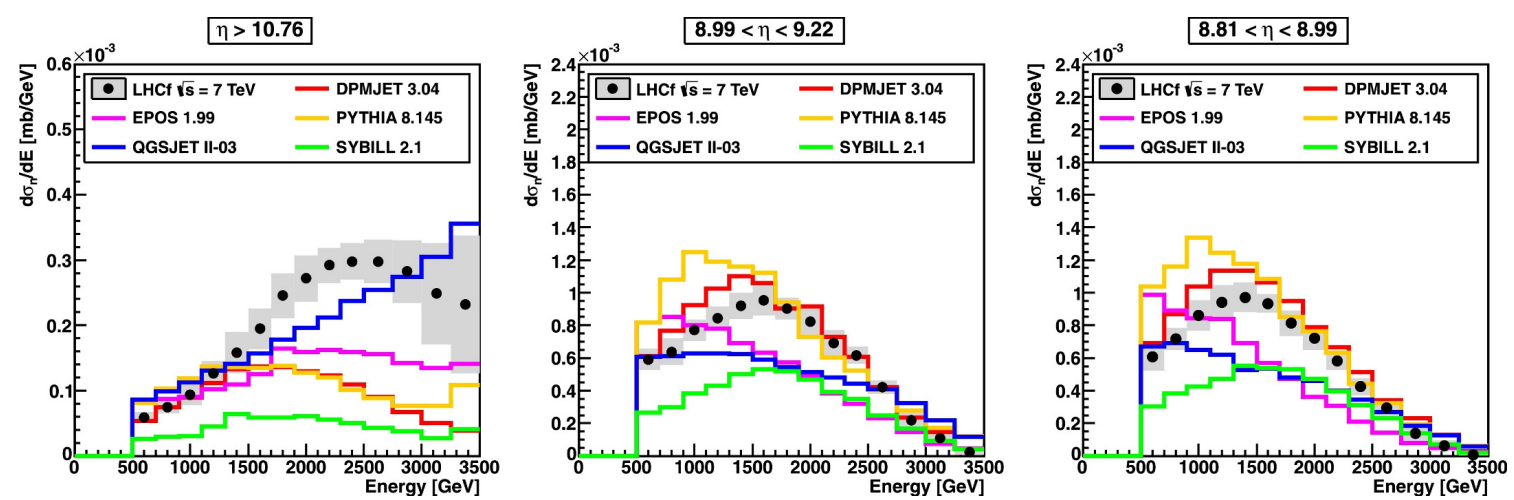

Figure 1: (color online). Neutron production spectra: comparison of the LHCf results with model predictions in different pseudo-rapidity regions. The black markers and gray shaded areas show the Arm1-Arm2 combined measurements and their systematic errors, respectively [7].

Among different properties that can be investigated by the experiment, forward production spectra are the main analysis objective of LHCf. The two important parameters in the development of EASs related to the forward region are the hardness of the energy spectra and indirectly the inelasticity $k$, defined in such a way that $1-k$ is the fraction of the energy of the primary particle carried out by the forward leading baryon. No model reproduces well the experimental data in all the regions considered in the analysis of $\gamma_{S}[4], \pi^{0} s[5,6]$ and neutrons [7]. In particular, the deviation observed for neutrons in the region $\eta>10.76$ is very strong: this is shown in Fig.1, where we can see the energy spectra of neutrons produced by p-p collisions at $\sqrt{s}=7 \mathrm{TeV}$ after Bayesian unfolding [8]. The high energy neutrons production rate observed by LHCf - and only qualitatively reproduced by the QGSJet II-03 model [9] - implies that inelasticity in the region $\eta>10.76$ is much lower than the one predicted by most models.

Aside from production spectra, there are at least two other points observable by LHCf that can provide important information for cosmic rays physics. The first one is the test of scaling laws $\left(<\mathrm{p}_{\mathrm{T}}>\right.$ scaling [10], Feynman scaling [11], limiting fragmentation [12, 13, 14]) that allow us to extrapolate the spectra measured at LHC to higher energies. The second one is the change in the production spectra due to the number of nucleons of the target, a crucial point if we consider that most of EASs develop from p-N or p-O collisions, not p-p. Being LHCf detectors optimized for electromagnetic showers, $\pi^{0} \mathrm{~s}$, reconstructed from their decays in two $\gamma \mathrm{s}$, are the best probe to investigate these points. Fig. 2 shows the $\pi^{0}$ inclusive production cross section as a function of $x_{F}=$ $2 p_{Z} / \sqrt{s}$ at different collision energies [6]. The Feynman hypothesis, predicting the independence of $\left(x_{F} / \sigma_{\text {inel }}\right)\left(d \sigma / d x_{F}\right)$ from $\sqrt{s}$ for $x_{F}>0.2$, holds at the $20 \%$ level. Fig.3 shows the nuclear modification factor - defined as the ratio of the $\pi^{0}$ inclusive production cross section for $\mathrm{p}-\mathrm{Pb}$ at $\sqrt{s_{N N}}=5.02 \mathrm{TeV}$ to the same quantity extrapolated for $\mathrm{p}-\mathrm{p}$ at $\sqrt{s}=5.02 \mathrm{TeV}$ - as a function of $p_{T}$ and $y_{l a b}$ [6]. Given the large uncertainties, all models are in good agreement with LHCf data, predicting a strong suppression of the production rate in the case the target nucleus is $\mathrm{Pb}$. 


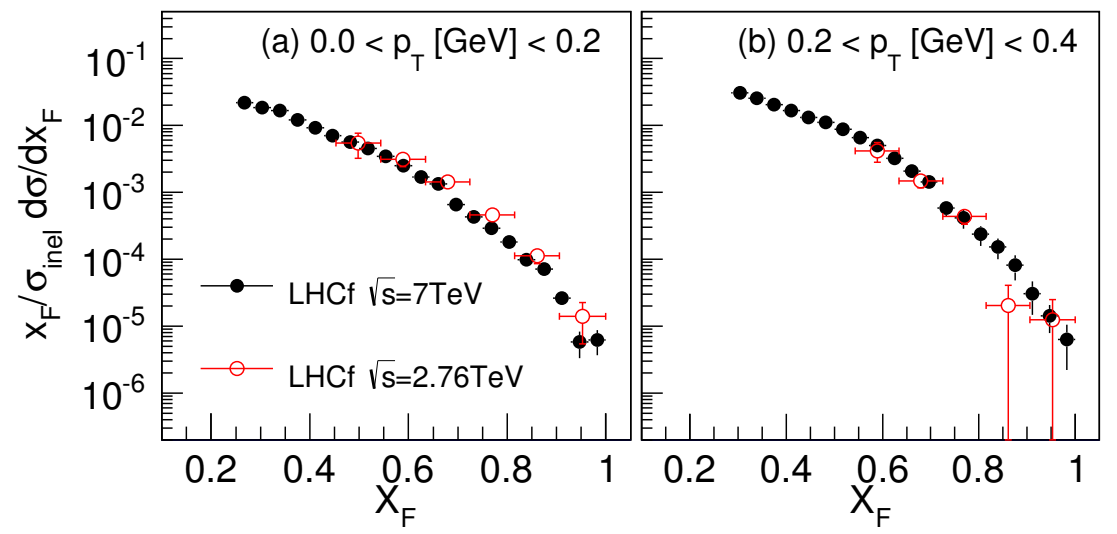

Figure 2: (color online). $\pi^{0}$ yield in each $p_{T}$ range as a function of $x_{F}$. Red open circles and black filled circles indicate measurements in p-p collisions at $\sqrt{s}=2.76$ and $7 \mathrm{TeV}$, respectively [6].
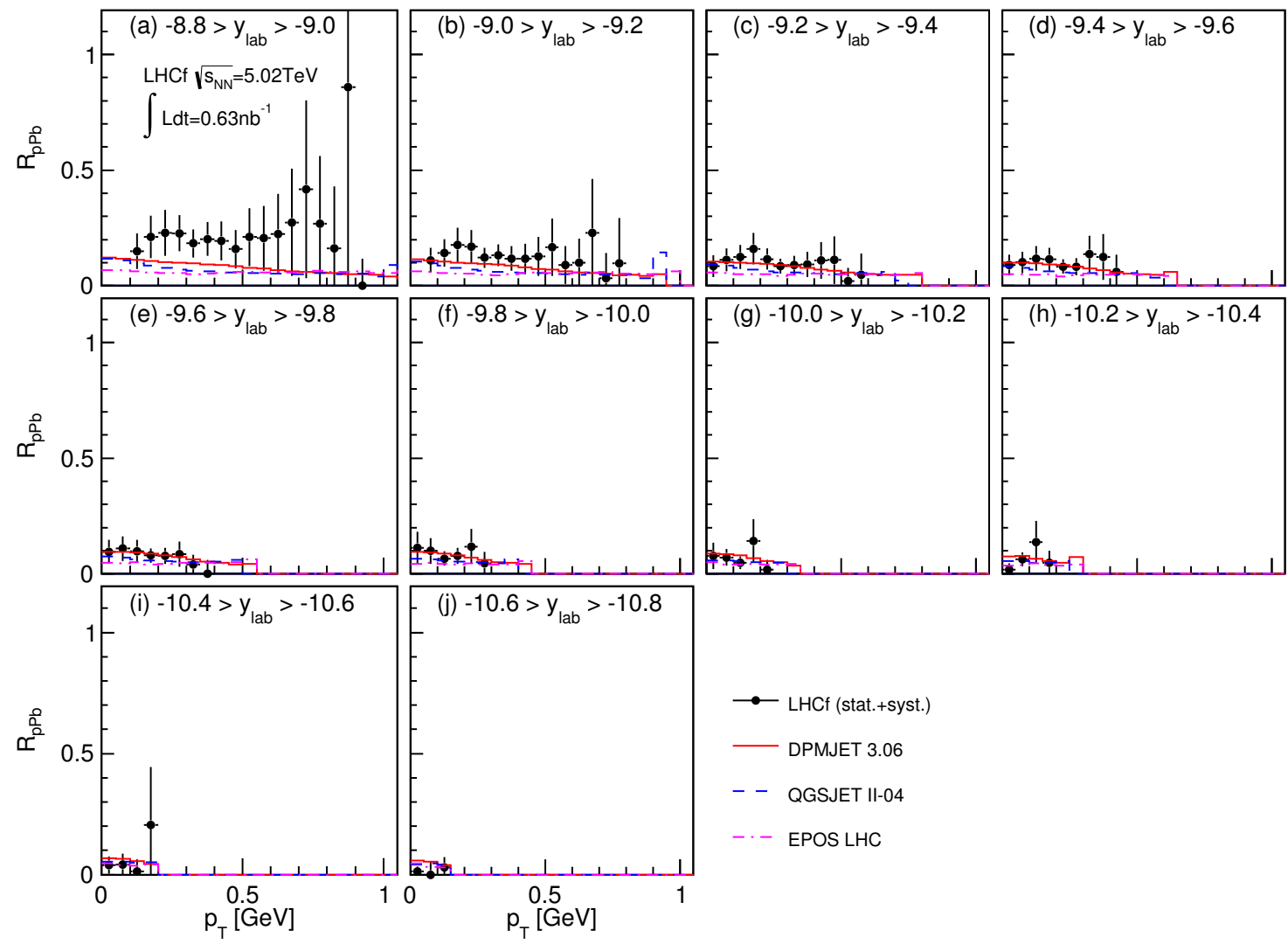

Figure 3: (color online). $\pi^{0}$ nuclear modification factor in each $p_{T}$ range as a function of $y_{l a b}$. Filled circles are LHCf measurements with the error bars incorporating both statistical and systematic uncertainties. Other lines are the predictions by hadronic interaction models [6]. 


\section{Conclusions and future prospects}

The LHCf experiment showed that in the forward region no model is perfectly reproducing experimental observations. Measurements of energy and $\mathrm{p}_{\mathrm{T}}$ spectra of the neutral particles produced in the very forward region can therefore be used to tune these models. New results at higher energies are expected from the ongoing analysis of data relative to p-p collisions at $\sqrt{s}=13 \mathrm{TeV}$ and $\mathrm{p}-\mathrm{Pb}$ collisions at $\sqrt{s_{N N}}=8.1 \mathrm{TeV}$. These last two data sets were acquired using a common trigger with the ATLAS experiment, so that we can combine the information of both detectors to identify different kinds of events, e.g. diffractive and non-diffractive. Moreover, in 2017 LHCf will acquire data from $\sqrt{s}=510 \mathrm{GeV}$ polarized p-p collisions at the Relativistic Heavy Ion Collider (RHIC) [16] in order to extend the $\sqrt{s}$ coverage for the test of Feynman scaling hypothesis.

\section{References}

[1] O. Adriani et al., JINST 3 (2008) S08006.

[2] T. Mase et al., Nucl.Instrum.Meth. A671 (2012) 29-136.

[3] K. Kawade et al., JINST 9 (2014) P03016 [hep-ex/1312 . 5950 ].

[4] O. Adriani et al., Physics Letters B 2 (2011) 128-134 [hep-ex/1104 . 5294].

[5] O. Adriani et al., Physical Review D 9 (2012) 092001 [hep-ex/205 . 4578].

[6] O. Adriani et al., Physical Review D 94 (2016) 032007 [hep-ex/1507 . 08764].

[7] O. Adriani et al., Physics Letters B 750 (2015) 360-366 [hep-ex/1503 . 03505].

[8] G. D’ Agostini, Nucl. Instrum. Meth A362 (1995) 487.

[9] S. Ostapchenko, Physical Review D 83 (2011) 014018.

[10] D. Amati, A. Stanghellini, S. Fubini et al., Il Nuovo Cimento 26 (1962) 896-954.

[11] R. Feynman, Physical Review Letters 23 (1969) 1415.

[12] J. Benecke, T.T. Chou, C.N. Yang, E. Yen, Physical Review 188 (1969) 2159-2169.

[13] J. Jalilian-Marian, Physical Review C 70 (2004) 027902.

[14] F. Gelis, A.M. Staśto, R. Venugopalan, Eur. Phys. J. C 48 (2006) 489-500.

[15] O. Adriani et al., Physical Review C 89 (2014) 065209 [hep-ex/1403. 7845 ].

[16] M. Harrison, T. Ludlam, S. Ozaki, Nucl.Instrum.Meth. A499 (2003) 235-244. 